\title{
Low Energy Gamma Radiation Induced Effects on Ultrasonic Velocity and Acoustic Parameters in Polyvinylidene Fluoride Solution
}

\author{
S. S. Kulkarni ${ }^{1}$ and U. V. Khadke ${ }^{2}$ \\ ${ }^{1}$ Department of Physics, KLS Gogte Institute of Technology, Belgaum, Karnataka, India \\ ${ }^{2}$ Department of Physics, Bheemanna Khandre Institute of Technology, Bhalki, India \\ Correspondence should be addressed to S. S. Kulkarni; sskphy@git.edu
}

Received 30 April 2016; Revised 4 August 2016; Accepted 18 August 2016

Academic Editor: Victor M. Castaño

Copyright (C) 2016 S. S. Kulkarni and U. V. Khadke. This is an open access article distributed under the Creative Commons Attribution License, which permits unrestricted use, distribution, and reproduction in any medium, provided the original work is properly cited.

\begin{abstract}
The modification of polyvinylidene fluoride (PVDF) polymer properties with irradiation is of interest as it possesses unique piezo-, pyro-, and ferroelectric properties. In this paper, we report the results of acoustic parameters of irradiated PVDF mixed with dimethylacetamide (DMAC) solution with low energy $\gamma$-source (Cs-137). The polymer solution covered with mica film assures only $\gamma$-ray passage and the duration was increased from 18 to 50 hours to achieve the higher dose rate. The dose rate was estimated using the strength of the radioactive source and the duration of the exposure. The ultrasonic velocity $(v)$, density $(\rho)$, and viscosity $(\eta)$ of $0.2 \mathrm{wt} \%$ and $0.5 \mathrm{wt} \%$ PVDF dissolved in pure DMAC solution, irradiated with different dose rate were measured using ultrasonic interferometer (Mittal make), Pyknometer, and Oswald's viscometer, respectively. It is observed that the values of $v$, $\rho$, and $\eta$ change with dose rate. The acoustic parameters such as adiabatic compressibility $(\beta)$, intermolecular free path length $\left(L_{f}\right)$, acoustic impedance $(Z)$, relative association (RA), ultrasonic attenuation $\left(\alpha / f^{2}\right)$, and relaxation time $(\tau)$ are calculated using the experimental data. These results are interpreted in terms of the solute-solvent interaction in a polymer solution and scissoring chain damage.
\end{abstract}

\section{Introduction}

Polyvinylidene fluoride (PVDF) is a semicrystalline polymer which has a simple chemical structure $\left[-\left(\mathrm{CH}_{2}-\mathrm{CF}_{2}\right)-\right]_{n}$ that has excellent mechanical and chemical properties with thermal stability. PVDF polymer exists in four crystalline forms, namely, $\alpha, \beta, \gamma$, and $\delta$, depending upon preparation conditions $[1,2]$. PVDF has been widely studied due to its piezo-, pyro-, and ferroelectric characteristics. The piezoelectric coefficients of uniaxial film are $d_{31}=21.4 \mathrm{Pc} / \mathrm{N}$, $d_{32}=2.3 \mathrm{Pc} / \mathrm{N}$, and $d_{33}=-31.5 \mathrm{Pc} / \mathrm{N}$ whereas pyroelectric coefficients are $-2.74 \cdot 10^{-5} \mathrm{C} / \mathrm{m}^{2} \mathrm{~K}$ [3]. These properties are useful for device application such as ultrasound transducers, nonvolatile memory, sensors, actuators, sonar instruments, and solar cells. The application of PVDF in the industries depends upon mechanical and thermal properties of PVDF.

The solvents used to dissolve PVDF play an important role and its prominent reactions are often overlooked.
Different solvents give rise to different rates, but changing a solvent can also alter the outcome of the reaction entirely. Most organic reactions are carried out in solution, although in some cases it is possible to simply mix all reacting components together. The specific choice of solvent can lead to the difference between success and failure in a particular reaction; therefore, an understanding polar aprotic solvent effect can be used productively to influence reactivity which is extensively used in the chemical, pharmaceutical, polymer processing and industries.

Radiation is an effective tool to modify the properties of any polymer. The modification may be in the form of crosslinking or main chain scission and molecular rearrangement. The radiation-induced structural changes in PVDF at two different temperatures were studied using XPS and reported [4]. They found that for PVDF irradiated at $150^{\circ} \mathrm{C}$, double bonds were formed due to dehydrofluorination of crosslinked molecules and stable structures of the samples 
irradiated at $20^{\circ} \mathrm{C}$. The electron beam irradiation PVDF shows decrease in its melting temperature with increase in dose rate. The gamma irradiation $\alpha$-PVDF sample changes to $\beta$ and $\gamma$ phases [5-7]. This indicates that the irradiation plays an important role in modification of the structure that may help to modify the properties of the sample. The irradiation effect on the properties of solid PVDF sample is considered but no work on solution sample and its properties is available in the literature. Hence, we have made an attempt to study the effect of low dose gamma radiation on polar polymer solutions. The changes in the acoustic properties are contributed to the formation of intermolecular crosslinking.

Ultrasonic studies in polymeric solutions have drawn the attention of many researchers in the recent years [8-12]. The extensive use of polymeric materials in technology has necessitated investigations of the molecular interactions of polymers and solvents [13-19]. Hence, in our experimental investigation, we used the ultrasonic technique to find the acoustic parameters such as adiabatic compressibility $(\beta)$, intermolecular free path length $\left(L_{f}\right)$, acoustic impedance $(Z)$, relative association (RA), ultrasonic attenuation $\left(\alpha / f^{2}\right)$, and relaxation time $(\tau)$ to correlate with the physical properties. All the parameters depend upon the structure of the polymer and nature of the solution.

\section{Materials and Methods}

Ferroelectric polymer PVDF of AR grade (99.8\% pure) in the powder form is procured from Sigma Aldrich. This PVDF powder is mixed with different concentrations of dimethylacetamide (AR grade 99.5\%) procured from HiMedia Laboratories Pvt. Ltd., India. The solution was magnetically stirred for 4 hours, so that the powder dissolves completely. The effect of $\gamma$-radiation on the prepared solutions was studied using the Cs-137 radioactive source. The acoustic properties are calculated for the irradiated solution. Effective variations in the physical parameters of polymer solutions due to high energy gamma irradiation have been reported in literature. Hence we have made an attempt to study the effect of low dose gamma radiation on PVDF solution as the radioactive source with low strength can create the permanent change in the molecular structure of the sample and also it is easily available.

2.1. Experimental Technique. There are many methods of polymer modifications that have been reported but other methods involve accurate stoichiometric mixture to attain particular modification that is led by complex structure of experiment such as usage of chemicals and formation of reactions. Hence, radiation method easily tunes the properties without any change in stoichiometric ratio of chemicals and the modification of the properties of polymer is very convenient. Experiment is carried out at Nuclear Science Lab, G. S. Science College, Belagavi, Karnataka, India, using Cs-137 radioactive source with the strength of $5 \mu \mathrm{Ci}$. The PVDF solution was irradiated by keeping the radioactive source on the solution sample in vacuum desiccators over a period of 18 to $50 \mathrm{hrs}$ to achieve considerable dose rate and to bring out any changes in the solution. Dose rate is estimated with the dose absorbed in unit time and indicates the amount of radioactive dose received. This was estimated using the strength of the radioactive source and duration of the exposure.

Ultrasonic interferometer, specific gravity bottle, and Ostwald viscometer are used to measure ultrasonic velocity, density, and viscosity, respectively. Ultrasonic velocity was measured using a single crystal interferometer (Mittal Enterprises, New Delhi) operating at $1 \mathrm{MHz}$ with an accuracy of $\pm 1.0 \mathrm{~m} / \mathrm{s}$. Densities of the solutions were measured using a $10 \mathrm{~mL}$ specific gravity bottle and the weight of the liquid was measured using an electronic balance (Model Shimadzu AX200). Accuracy in the measurement of densities of the solutions is $\pm 0.1 \mathrm{~kg} / \mathrm{m}^{3}$. The viscosity was measured with an Ostwald's Viscometer and the flow time with a digital stopwatch. The time flow is measured using digital stop watch capable of registering time accurate to $\pm 0.1 \mathrm{~s}$. Measurements were repeated thrice to confirm the accuracy of results.

The acoustic parameters were computed as follows.

The ultrasonic velocity is calculated as

$$
v=f \lambda,
$$

where " $f$ " is the frequency of the ultrasonic waves and " $\lambda$ " is the measured wavelength value of ultrasonic waves in a given solution.

The viscosity of the solution is calculated using

$$
\eta=\frac{d_{l} t_{l}}{d_{w} t_{w}} \eta_{w},
$$

where $d_{l}$ and $t_{l}$ are density and time flow of liquid, whereas $d_{w}$ and $t_{w}$ are density and time flow of water. The density of the solution is taken as mass per unit volume.

The following equations are used to compute the acoustic parameters: adiabatic compressibility $(\beta)$, intermolecular free path length $\left(L_{f}\right)$, acoustic impedance $(Z)$, relative association (RA), ultrasonic attenuation $\left(\alpha / f^{2}\right)$, and relaxation time $(\tau)$.

\subsection{Theoretical Calculations}

(1) Adiabatic compressibility $(\beta)$ has been calculated from the ultrasonic velocity " $v$ " and the density " $\rho$ " of the solution using the Newton-Laplace equation [20]:

$$
\beta=\frac{1}{\rho v^{2}}
$$

(2) Intermolecular free path length $\left(L_{f}\right)$ has been determined as follows [21]:

$$
L_{f}=K \beta^{1 / 2}
$$

where $K$ is the temperature dependent Jacobson's constant $\left(K=205.35 \times 10^{-8}\right.$ at $\left.300 \mathrm{~K}\right)$ and $\beta$ is the adiabatic compressibility.

(3) Acoustic impedance $(Z)$ is given as follows:

$$
Z=\rho v,
$$


TABLE 1: Variation of density, viscosity, and ultrasonic velocity and acoustic parameters of PVDF with different concentration in dimethylacetamide.

\begin{tabular}{lcccc}
\hline $\begin{array}{l}\text { Concentration “C” } \\
\text { mass\% }\end{array}$ & $\begin{array}{c}\text { Density “ } \rho \text { " } \\
\mathrm{kg} / \mathrm{m}^{3}\end{array}$ & $\begin{array}{c}\text { Viscosity “ } \eta \text { ” } \\
\mathrm{Nsm}^{-2} \times 10^{-3}\end{array}$ & $\begin{array}{c}\text { Ultrasonic velocity “ } v \text { ” } \\
\mathrm{m} / \mathrm{s}\end{array}$ & $\begin{array}{c}\text { Compressibility “ } \beta \text { ” } \\
\times 10^{-10}\end{array}$ \\
\hline 0.00 & 949.730 & 0.866 & 1454.00 & 4.980 \\
0.20 & 957.620 & 1.113 & 1488.30 & 4.714 \\
0.50 & 1116.690 & 1.337 & 1510.00 & 3.927 \\
\hline
\end{tabular}

TABLE 2: Variation of acoustic parameters: compressibility, intermolecular free path length, acoustic impedance, relative association, attenuation, and relaxation time of PVDF with different concentration in dimethylacetamide.

\begin{tabular}{lccccc}
\hline $\begin{array}{l}\text { Concentration " } C \text { " } \\
\text { mass\% }\end{array}$ & $\begin{array}{c}\text { Intermolecular free } \\
\text { path length " } L \text { " } \\
\times 10^{-11}\end{array}$ & $\begin{array}{c}\text { Acoustic impedance } \\
\text { " } Z \text { ” } \\
\times 10^{6}\end{array}$ & $\begin{array}{c}\text { Relative } \\
\text { association (RA) }\end{array}$ & $\begin{array}{c}\text { Ultrasonic } \\
\text { attenuation } \\
\times 10^{-14}\end{array}$ & $\begin{array}{c}\text { Relaxation time } \\
10^{-13}\end{array}$ \\
\hline 0.00 & 4.5827 & 1.3809 & - & 2.34544 & 5.75739 \\
0.20 & 4.4586 & 1.4252 & 0.9965 & 2.7859 & 6.9999 \\
0.50 & 4.0695 & 1.6862 & 0.9950 & 2.7476 & 7.0045 \\
\hline
\end{tabular}

where $\rho$ and $v$ are the density and velocity of the solution, respectively.

(4) Relative association (RA) is given as follows:

$$
\mathrm{RA}=\left(\frac{\rho}{\rho_{o}}\right)\left(\frac{v_{o}}{v}\right)
$$

where $\rho_{o}$ and $v_{o}$ are the density and velocity of the solvent.

(5) Ultrasonic attenuation $\left(\alpha / f^{2}\right)$ and relaxation time $(\tau)$ were calculated using the following [22]:

$$
\begin{aligned}
\frac{\alpha}{f^{2}} & =\frac{8 \pi^{2} \eta}{\rho v^{3}}, \\
\tau & =\frac{4 \eta}{3 \rho v^{2}},
\end{aligned}
$$

where $\eta$ is the viscosity of the solution.

(6) Dose rate $=($ source strength $) \times($ duration of exposure).

\section{Results and Discussion}

The experimental measurements of density, viscosity, and velocity of ultrasonic waves at room temperature $(300 \mathrm{~K})$ for two different concentrations of PVDF solutions are shown in Table 1. The estimated values of acoustic parameters from experimental data are shown in Table 2. The PVDF polymer solution of pure, $0.2 \mathrm{wt} \%$, and $0.5 \mathrm{wt} \%$ was irradiated with Cs137 source for different dose rates which are shown in Tables 3 and 4, respectively. Figure 1 shows the X-ray diffraction pattern of the procured PVDF. The diffraction peak observed at $2 \theta=18.09^{\circ}, 19.58^{\circ}$ confirms the formation of $\beta$ phase of the powder sample [23]. The variation of relative association, acoustic impendence, compressibility, intermolecular free path length, relaxation time, and ultrasonic attenuation with dose rate for two different concentrations is plotted in Figures $2,3,4,5,6$, and 7 , respectively.

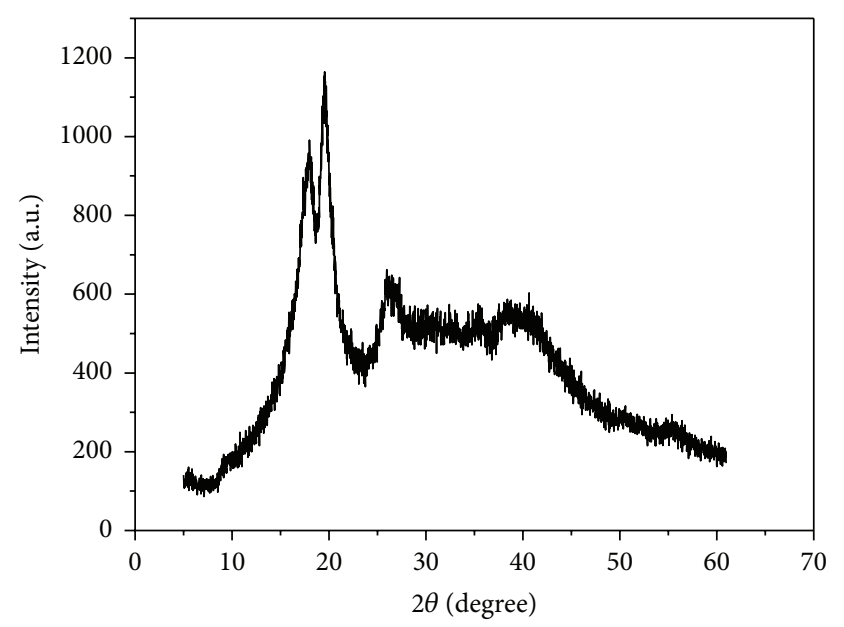

FIGURE 1: XRD pattern of virgin PVDF powder confirming the phase formation.

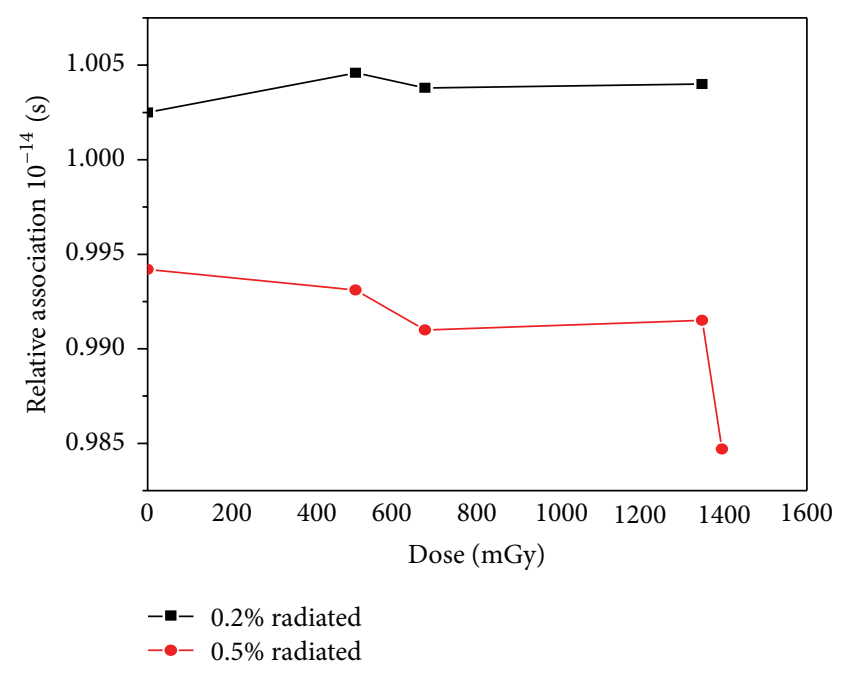

FIGURE 2: Variation of relative association of $0.2 \%$ and $0.5 \%$ PVDF solution with dose rate. 


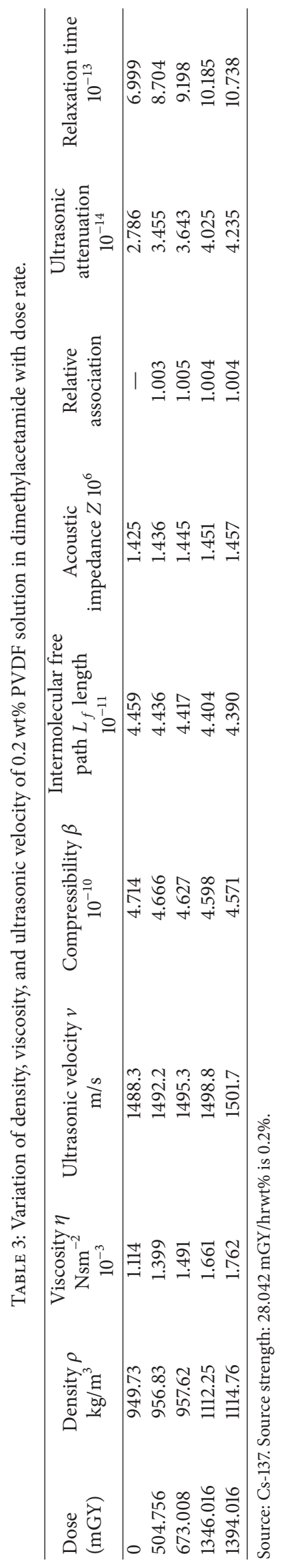




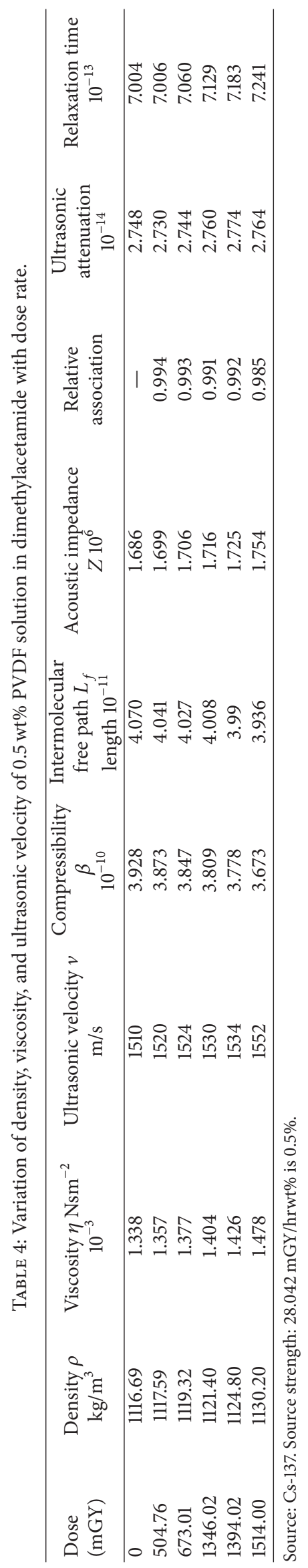




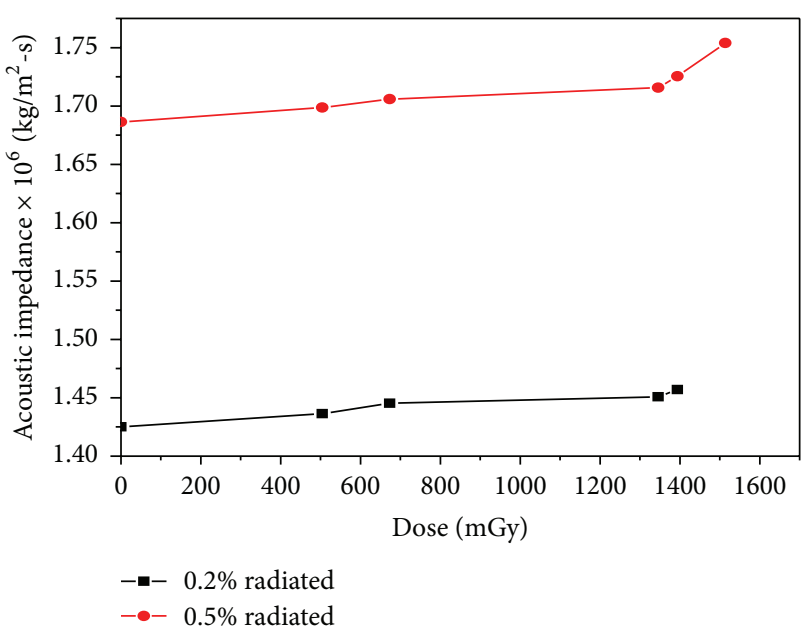

FIGURE 3: Variation of acoustic impedance of $0.2 \%$ and $0.5 \%$ PVDF solution with dose rate.

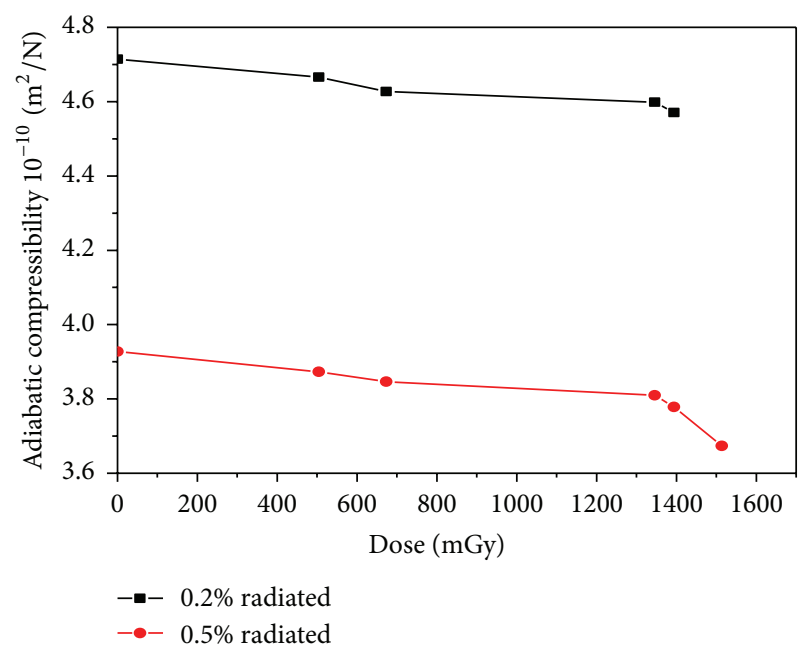

FIGURE 4: Variation of adiabatic compressibility of $0.2 \%$ and $0.5 \%$ PVDF solution with dose rate.

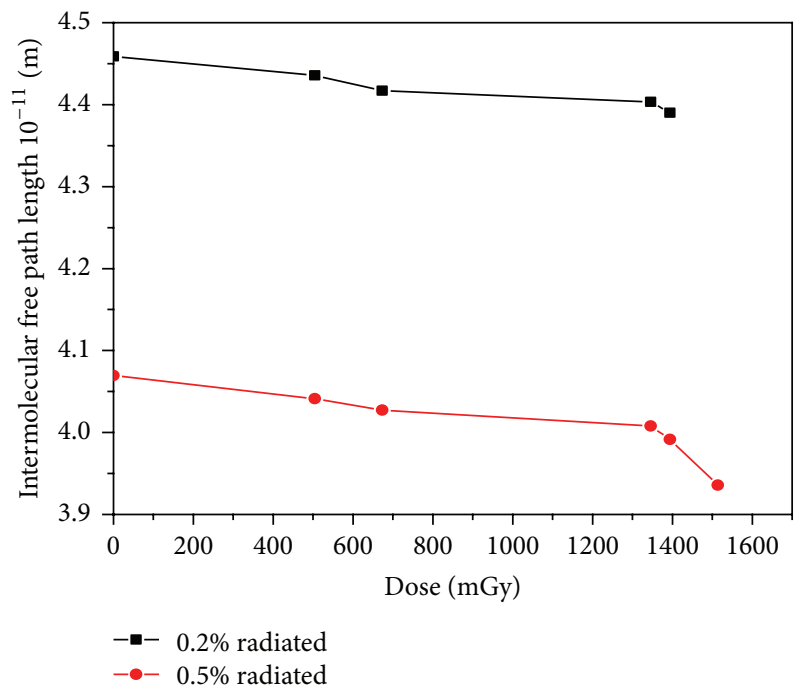

FIGURE 5: Variation of intermolecular free path length of $0.2 \%$ and $0.5 \%$ PVDF solution with dose rate.

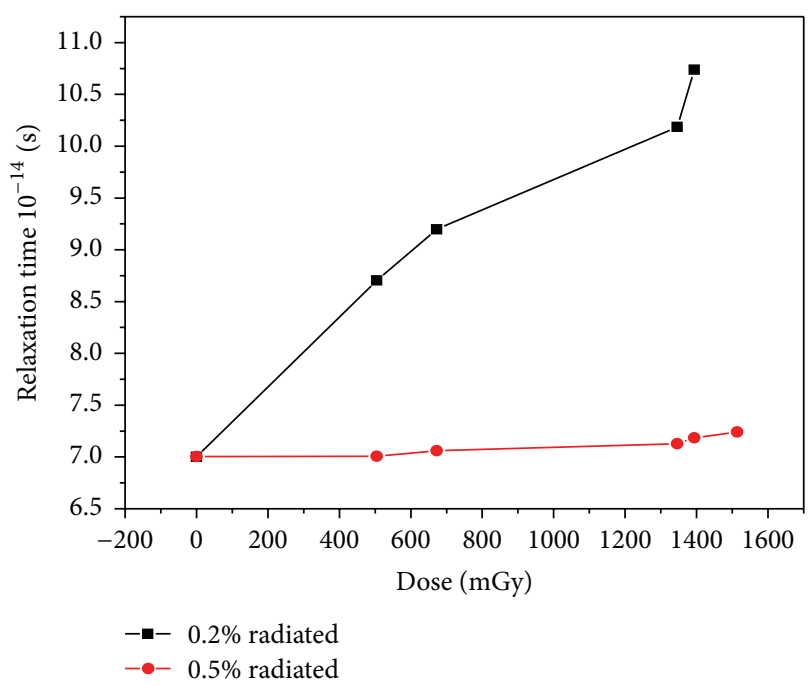

FIGURE 6: Variation of relaxation time of $0.2 \%$ and $0.5 \%$ PVDF solution with dose rate.

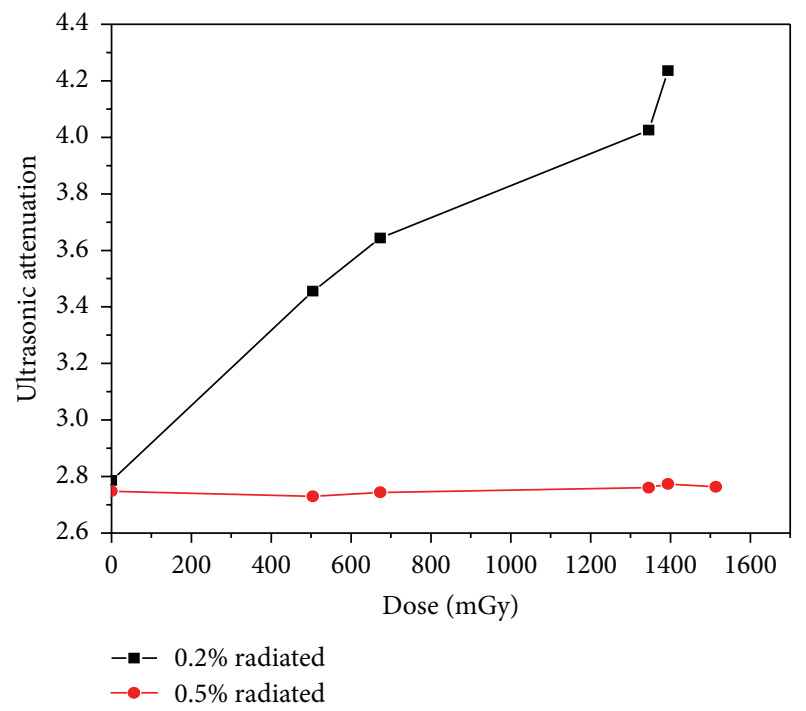

FIgURE 7: Variation of ultrasonic attenuation of $0.2 \%$ and $0.5 \%$ PVDF solution with dose rate.

It was found that $v, \rho$, and $\eta$ increase with increase in dose rate suggesting that polymer may undergo chain scission. As the concentration of PVDF increases the number of intermolecules crosslinking in the medium increases which leads to lesser compressibility and hence ultrasonic velocity increases. Further the number of particles increases the frictional resistance between the layers of medium. Because of radiation, the molecule shrinks and the increase in the viscosity is due to increase in the molecular weight of the solution with dose rate.

In order to understand the effect of low energy $\gamma$ irradiation on PVDF polymeric solution, the acoustic parameters such as adiabatic compressibility $(\beta)$, intermolecular free path length $\left(L_{f}\right)$, acoustic impedance $(Z)$, relative association (RA), ultrasonic attenuation $\left(\alpha / f^{2}\right)$, and relaxation 
time $(\tau)$ were determined using standard equations and are correlated with concentration and dose rate. The relative association, acoustic impedance, adiabatic compressibility, and intermolecular free path length show any changes with increase in dose rate as shown in Figures 2, 3, 4, and 5, respectively.

The intermolecular free path length $\left(L_{f}\right)$ and the compressibility $(\beta)$ of PVDF solution show decreasing trend at higher dose rate. Decrease in $L_{f}$ with dose rate supports solvent-solute interactions. The acoustic impendence of the solution depends on inertial and elastic properties of the medium and also on concentration. Hence it is observed that the increase of acoustic impendence at higher dose rate is almost linear. Because acoustic impendence depends on ultrasonic velocity and density. With increase in dose, both parameters ultrasonic velocity and density will increase. However, for PVDF solution of $0.2 \mathrm{wt} \%$, the relaxation time and ultrasonic attenuation show increasing trend of almost $50 \%$ at higher dose rate and become subsidized at higher concentration of PVDF as shown in Figures 6 and 7, respectively.

The relaxation time increases linearly with concentration. Further it is found that relaxation time $(\tau)$ for $0.2 \mathrm{wt} \%$ solution increases with dose rate whereas the relaxation time $(\tau)$ for $0.5 \mathrm{wt} \%$ solution is almost saturated with dose rate and that is indicative of modification of structural properties of the $0.2 \mathrm{wt} \%$ PVDF solution.

The variation of ultrasonic velocity in a solution depends on the intermolecular free length on mixing. On the basis of a model for sound propagation proposed by Kincaid and Eyring [24] ultrasonic velocity increases on decrease of free path length and vice versa.

The property which can be studied to understand the interaction is relative association (RA). It can be explained by two factors: (1) the breaking up of solvent molecules on addition of solute to it and (2) solvation of the solute molecule. The former leads to decrease and the latter to the increase of relative association. In our study the values of RA increases with increase in the solute concentration due to solvation of the molecule relative association (RA).

\section{Conclusion}

Ultrasonic velocity $(v)$, density $(\rho)$, and viscosity $(\eta)$ of pure and irradiated solutions are measured using ultrasonic interferometer (Mittal make), Pyknometer and Oswald's viscometer, respectively. The PVDF solution of $0.2 \mathrm{wt} \%$ shows the variation in ultrasonic attenuation and relaxation time at higher dose rate and saturates at and above $0.5 \mathrm{wt} \% \mathrm{PVDF}$ solution. The saturation of attenuation of ultrasound propagation in PVDF solution at and above $0.5 \mathrm{wt} \%$ is contributed to the stability in the relaxation time of the molecules. The relative association, compressibility, acoustic impedance, and the intermolecular free path length remains unchanged with dose rate.

\section{Competing Interests}

The authors declare that they have no competing interests.

\section{References}

[1] H. Kawai, "The piezoelectricity of poly (vinylidene Fluoride)," Japanese Journal of Applied Physics, vol. 8, no. 7, pp. 975-976, 1969.

[2] R. G. Kepler and R. A. Anderson, "Ferroelectricity in polyvinylidene fluoride," Journal of Applied Physics, vol. 49, no. 3, pp. 1232-1235, 1978.

[3] K. Tashiro and H. S. Nalwa, Ferroelectric Polymers, Marcel Dekker, New York, NY, USA, 1995.

[4] X. Zhong, L. Yu, W. Zhao, Y. Zhang, and J. Sun, "XPS studies of radiation-induced structural changes in polyvinylidene fluoride," Polymer Degradation and Stability, vol. 39, no. 3, pp. 399402, 1993.

[5] Y. M. Lim, P. H. Kang, S. M. Lee et al., "Effect of electron beamirradiation on poly(vinylidene fluoride) films at the melting temperature," Journal of Industrial and Engineering Chemistry, vol. 12, no. 4, pp. 589-593, 2006.

[6] S. Aarya, Siddhartha, A. K. Srivastava, A. Saha, and W. A. Wahab, "Effect of $1.25 \mathrm{MeV}$ gamma irradiation in $\alpha$-phased PVDF," Nuclear Instruments and Methods in Physics Research, Section B: Beam Interactions with Materials and Atoms, vol. 267, no. 21-22, pp. 3545-3548, 2009.

[7] E. Adem, J. Rickards, G. Burillo, and M. Avalos-Borja, "Changes in poly-vinylidene fluoride produced by electron irradiation," Radiation Physics and Chemistry, vol. 54, no. 6, pp. 637-641, 1999.

[8] K. Sreekanth, M. Kondaiah, D. Sravana Kumar, and D. Krishna Rao, "Excess acoustical and volumetric properties and theoretical estimation of ultrasonic velocities in binary liquid mixtures of 2-chloroaniline with acrylic esters at $308.15 \mathrm{~K}$," Journal of Solution Chemistry, vol. 41, no. 7, pp. 1088-1102, 2012.

[9] M. K. Praharaj, A. Satapathy, P. Mishra, and S. Mishra, "Ultrasonic studies of ternary liquid mixtures of N-Ndimethylformamide, nitrobenzene, and cyclohexane at different frequencies at $318 \mathrm{~K}$," Journal of Theoretical and Applied Physics, vol. 7, article 23, 2013.

[10] B. Nagarjun, A. V. Sarma, G. V. R. Rao, and C. Rambabu, "Thermodynamic and acoustic study on molecular interactions in certain binary liquid systems involving ethyl benzoate," Journal of Thermodynamics, vol. 2013, Article ID 285796, 9 pages, 2013.

[11] D. R. Godhani, P. B. Dobariya, A. M. Sanghani, and J. P. Mehta, "Thermodynamic properties of binary mixtures of 1 , 3,4-oxadiazole derivative with chloroform, $N, N$-dimethyl formamide at 303, 308 and $313 \mathrm{~K}$ and atmospheric pressure," Arabian Journal of Chemistry, 2012.

[12] F. M. Sannaningannavar, B. S. Navati, and N. H. Ayachit, "Studies on thermo-acoustic parameters in the dilute solutions of poly(ethyleneglycol)," Polymer Bulletin, vol. 70, no. 2, pp. 603-618, 2013.

[13] K. P. Singh and S. C. Bhatt, "Investigation of acoustical parameters of polyvinyl acetate," Applied Physics Research, vol. 2, no. 1, pp. 35-45, 2010.

[14] P. J. Vasoya, N. M. Mehta, V. A. Patel, and P. H. Parsania, "Effect of temperature on ultrasonic velocity and thermodynamic parameters of cardo aromatic polysulfonate solutions," Journal of Scientific and Industrial Research, vol. 66, no. 10, pp. 841-848, 2007.

[15] S. K. Mehta, R. Jain, S. Sharma, and K. K. Bhasin, "Interaction of poly(ethylene glycol)-400 with tetraethylammonium bromide 
in aqueous media," Journal of Molecular Liquids, vol. 122, no. 13, pp. 15-20, 2005.

[16] V. Kannappan, B. S. Santhi, and D. R. Jonathan, "Ultrasonic investigation on segmental motion of some polymers in solution," Indian Journal of Chemistry A, vol. 53, no. 6, pp. 688-694, 2014.

[17] P. S. Nikam, B. S. Jagdale, A. B. Sawant, and M. Hassan, "Ultrasonic study of binary liquid mixtures of benzonitrile with alkanols (C 1C 3) at various temperatures," Acoustic Letters, vol. 22, pp. 199-203, 1999.

[18] S. S. Kulkarni and U. V. Khadke, "Effect of solvents on the ultrasonic velocity and acoustic parameters of polyvinylidene fluoride solutions," Indian Journal of Materials Science, vol. 2016, Article ID 9582582, 6 pages, 2016.

[19] U. G. Pathak, J. V. Patel, and P. H. Parsania, "Ultrasonic speed and thermodynamic parameters of novel epoxy resin solutions based on cardo symmetric double Schiff bases at $308.15 \mathrm{~K}$," Journal of Solution Chemistry, vol. 41, no. 5, pp. 755-765, 2012.

[20] D. N. Rao, A. Krishnaiah, and P. R. Naidu, "Excess thermodynamic properties of liquid ethylenediamine+anaromatichydrocarbon," Acta Chimica Academiae Scientiarum Hungaricae, vol. 107, no. 1, pp. 49-55, 1981.

[21] P. S. Nikam and M. Hasan, "Ultrasonic velocity and apparent molar compressibility of trichloroacetic acid in aqueous ethanol," Asian Journal of Chemistry, vol. 5, no. 2, pp. 319-321, 1993.

[22] J. K. Das, S. K. Dash, N. Swain, and B. B. Swain, "Ultrasonic investigation in a polar-polar system-Metyl Isobutyl Ketone (MIBK) and aliphatic alcohols," Journal of Molecular Liquids, vol. 81, no. 2, pp. 163-179, 1999.

[23] A. Tawansi, A. H. Oraby, E. M. Abdelrazek, and M. Abdelaziz, "Structural and electrical properties of $\mathrm{MgCl}_{2}$-filled PVDF films," Polymer Testing, vol. 18, no. 8, pp. 569-579, 1999.

[24] J. F. Kincaid and H. Eyring, "Free volumes and free angle ratios of molecules in liquids," The Journal of Chemical Physics, vol. 6, no. 10 , pp. 620-629, 1938. 

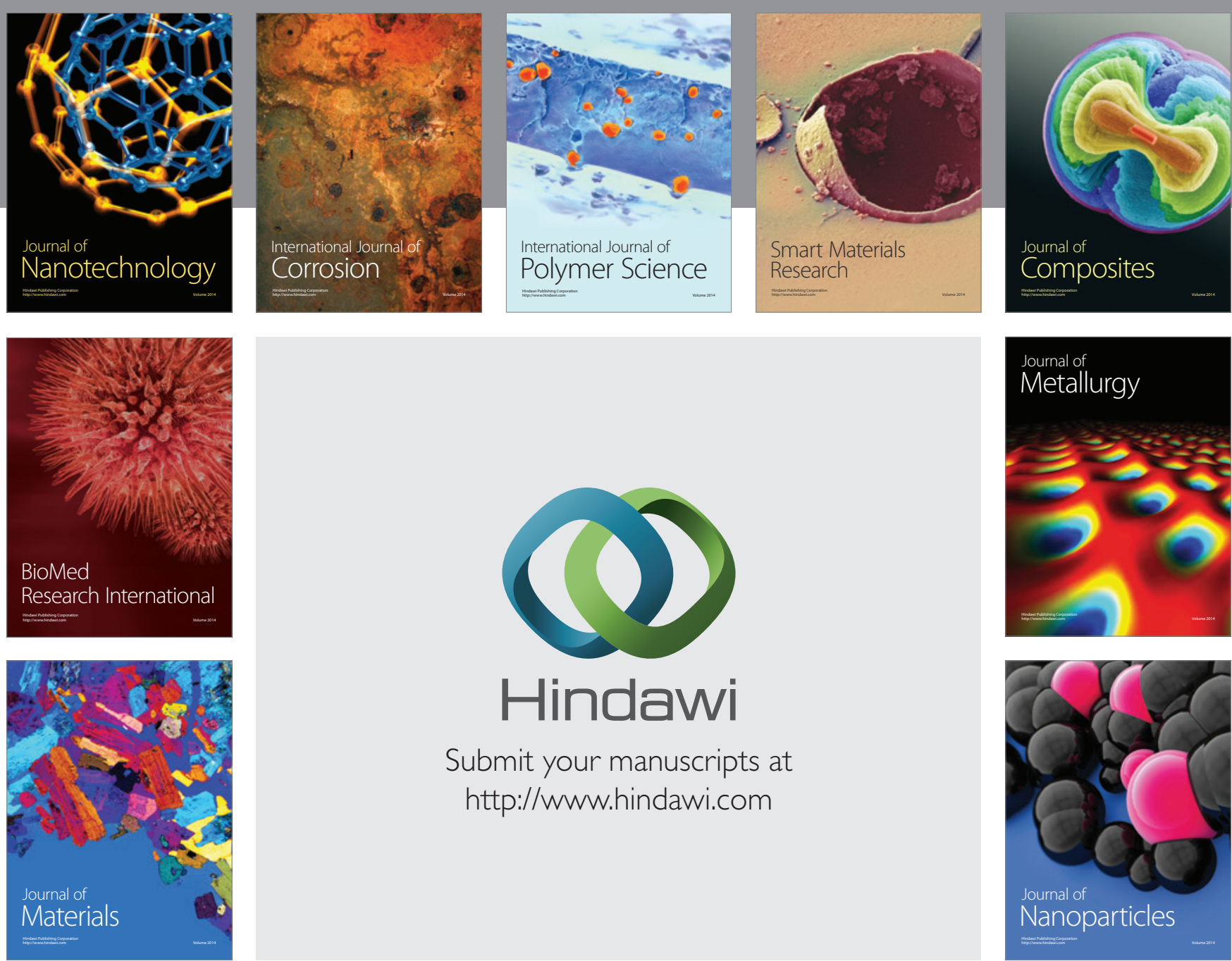

\section{Hindawi}

Submit your manuscripts at

http://www.hindawi.com

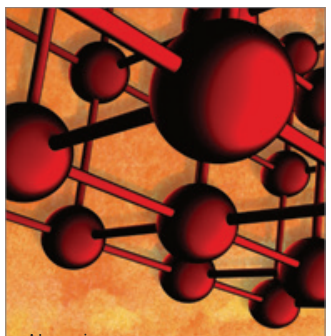

Materials Science and Engineering
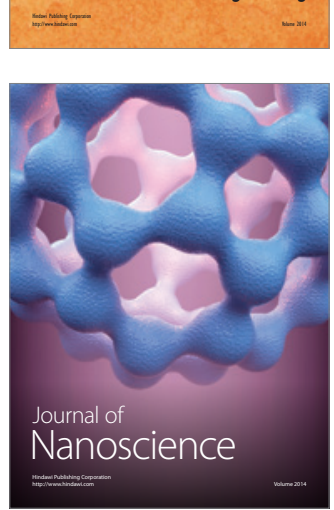
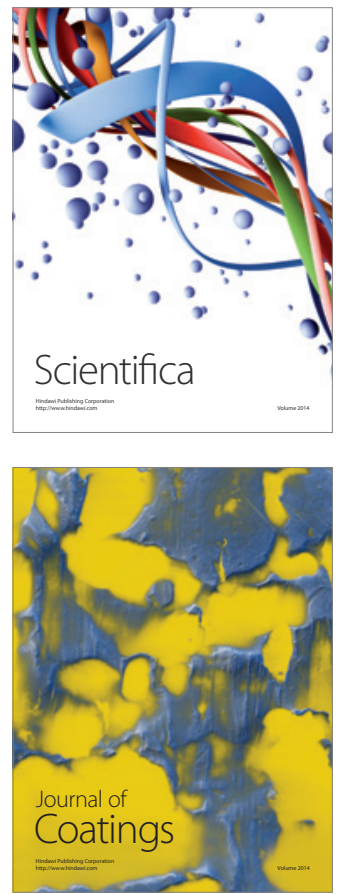
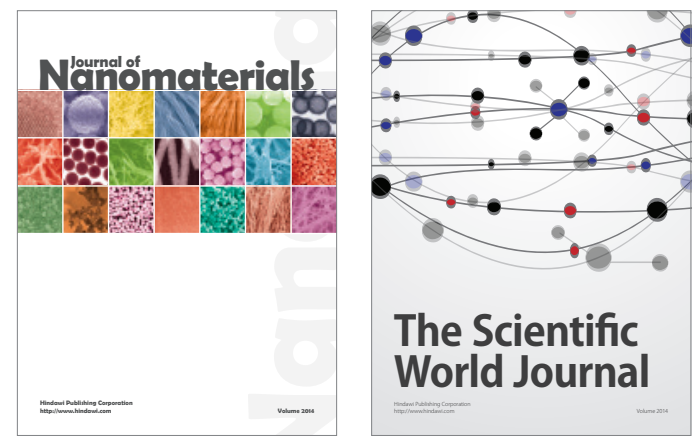

The Scientific World Journal
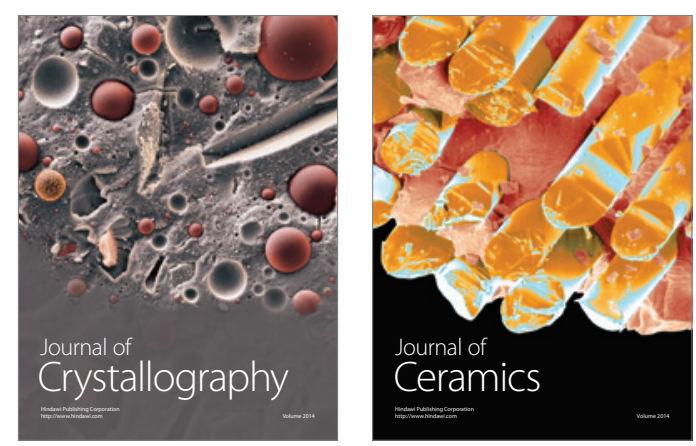
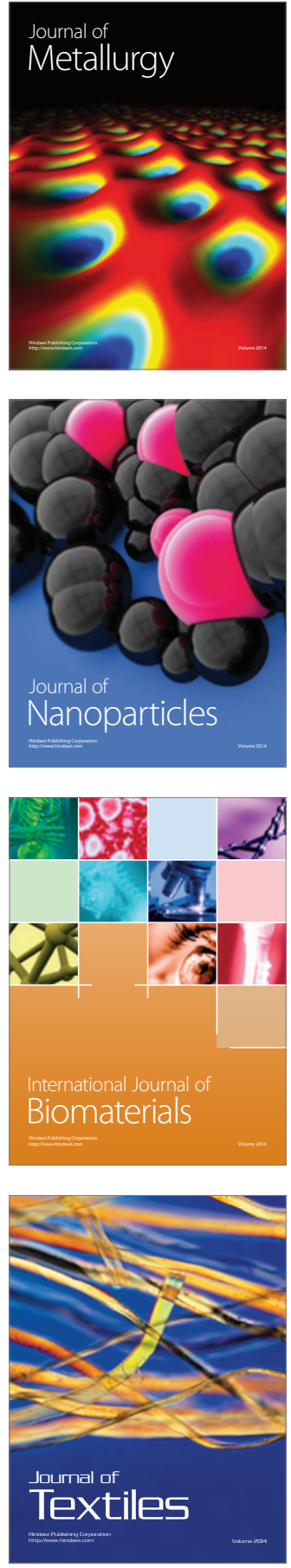